\title{
Two types of nonlinear wave equations for diffractive beams in bubbly liquids with nonuniform bubble number density
}

\author{
Tetsuya Kanagawa ${ }^{a)}$ \\ Department of Engineering Mechanics and Energy, Graduate School of Systems and Information Engineering, \\ University of Tsukuba, 1-1-1 Tennodai, Tsukuba 305-8573, Japan
}

(Received 30 January 2014; revised 9 March 2015; accepted 13 March 2015)

\begin{abstract}
This paper theoretically treats the weakly nonlinear propagation of diffracted sound beams in nonuniform bubbly liquids. The spatial distribution of the number density of the bubbles, initially in a quiescent state, is assumed to be a slowly varying function of the spatial coordinates; the amplitude of variation is assumed to be small compared to the mean number density. A previous derivation method of nonlinear wave equations for plane progressive waves in uniform bubbly liquids [Kanagawa, Yano, Watanabe, and Fujikawa (2010). J. Fluid Sci. Technol. 5(3), 351-369] is extended to handle quasi-plane beams in weakly nonuniform bubbly liquids. The diffraction effect is incorporated by adding a relation that scales the circular sound source diameter to the wavelength into the original set of scaling relations composed of nondimensional physical parameters. A set of basic equations for bubbly flows is composed of the averaged equations of mass and momentum, the Keller equation for bubble wall, and supplementary equations. As a result, two types of evolution equations, a nonlinear Schrödinger equation including dissipation, diffraction, and nonuniform effects for high-frequency short-wavelength case, and a Khokhlov-Zabolotskaya-Kuznetsov equation including dispersion and nonuniform effects for low-frequency long-wavelength case, are derived from the basic set.
\end{abstract}

(C) 2015 Acoustical Society of America. [http://dx.doi.org/10.1121/1.4916371]

Pages: 2642-2654

\section{INTRODUCTION}

Liquids containing gas bubbles exhibit fairly complex acoustic properties due to the complexity of gas-liquid twophase flow and a variety of wave properties (e.g., Nigmatulin, 1991; Nakoryakov et al., 1993; Yano et al., 2013). Weakly nonlinear analysis (e.g., Nayfeh, 1973; Jeffrey and Kawahara, 1982) is effective for evaluating the phenomena observed in such liquids because nonlinear (i.e., finite amplitude) effects appear during long-range propagation processes and can significantly alter the wave behavior, even for small wave amplitudes.

van Wijngaarden (1968) revealed that plane sinusoidal waves evolve into acoustic solitons (solitary waves) because of both the nonlinear effect and a dispersion effect induced by bubble oscillations. The derivation of the Korteweg-de Vries (KdV) equation predicted the formation of an acoustic soliton in a bubbly liquid (van Wijngaarden, 1968), but a later derivation of the KdV-Burgers (KdVB) equation (van Wijngaarden, 1972) incorporating a dissipation effect contradicted the existence of solitons because the dissipation effect that appears and competes with the nonlinear and dispersion effects. Thus, the propagation properties of weakly nonlinear waves in bubbly liquids depend on not only the nonlinear effect but also other cooperative effects such as the dispersion and dissipation. Although soliton formation (van Wijngaarden, 1968) was an important discovery, it is only one typical evolution of nonlinear waves in bubbly

\footnotetext{
a) Author to whom correspondence should be addressed. Electronic mail: kanagawa@kz.tsukuba.ac.jp
}

liquids. Correct wave equations are thereby essential for a sufficient understanding of the physics governing the wave behavior. A number of theoretical studies have intensively been performed (Kuznetsov et al., 1978; Commander and Prosperetti, 1989; Nigmatulin, 1991; Gumerov, 1992; Nakoryakov et al., 1993; Akhatov et al., 1996; Khismatullin and Akhatov, 2001; Liang et al., 2008; Vanhille and Campos-Pozuelo, 2009; Ando et al., 2011; Leroy et al., 2011; Grandjean et al., 2012; Louisnard, 2012; Kudryashov and Sinelshchikov, 2013, 2014, to name a few), and various nonlinear wave equations describing weakly nonlinear phenomena were submitted. The KdVB equation (e.g., van Wijngaarden, 1968, 1972) and the nonlinear Schrödinger (NLS) equation (e.g., Gumerov, 1992; Akhatov et al., 1996) are two well-known nonlinear equations for plane waves in uniform bubbly liquids. On the other hand, the Khokhlov-Zabolotskaya-Kuznetsov (KZK) equation (Zabolotskaya and Khokhlov, 1969; Kuznetsov, 1971; Hamilton, 1998) was derived to describe nonlinear sound beams (i.e., quasi-plane waves) in compressible Newtonian single-phase (not bubbly) flows. In real situation, however, we often observe quasi-plane waves in liquids containing nonuniformly distributing bubbles. For example, the medical applications have intensively been developed: (i) diagnosis utilizing nonuniformly distributed microbubbles as a contrast agent in the focused ultrasound imaging, (ii) treatment by the microbubble-enhanced focused ultrasound (e.g., Bailey et al., 2003). Numerical predictions for these applications are currently performed by using the nonlinear wave equation for single-phase (not two-phase) fluids. Therefore, generalized KZK and NLS equations (as derived here), which 
are based on the basic equations for gas-liquid two-phase flows, and which incorporate the diffraction effect as quasiplane waves and the nonuniformity of the bubble distribution, will progress the predictions for various problems on acoustics in bubbly liquids.

Kanagawa et al. (2010) (or Yano et al., 2013) proposed a unified method to derive nonlinear wave equations for plane wave propagation in uniform bubbly liquids. This method is based on a set of scaling relations among the physical parameters that are appropriate to a specific wave phenomenon, and parameter scaling is the measurement of the nondimensional magnitudes of physical quantities in terms of a typical nondimensional amplitude of the wave under consideration. Some examples of nondimensional magnitudes are expressed by the following ratios: the ratio of a typical bubble radius $R_{0}^{*}$ to a typical wavelength $L^{*}$, that of a typical frequency of the wave $\omega^{*}$ to an eigenfrequency of the bubble $\omega_{\mathrm{B}}^{*}$, and that of the typical propagation speed of the wave $U^{*}$ to the speed of sound in a liquid $c_{\mathrm{L} 0}^{*}$. In Fig. 1, the linear dispersion relation for waves in bubbly liquids neglecting the dissipation and liquid compressibility can be depicted conceptually (van Wijngaarden, 1968, 1972). Then, Kanagawa et al. (2010) clarified that the NLS equation including the dissipation effect for envelopes of high-frequency short-wavelength carrier wave and the KdVB equation for waves of low-frequency long-wavelength (Fig. 1) can be derived by applying the following scaling relations:

$$
\left(\frac{U^{*}}{c_{\mathrm{L} 0}^{*}}, \frac{\omega^{*}}{\omega_{\mathrm{B}}^{*}}, \frac{R_{0}^{*}}{L^{*}}\right) \equiv\left(O\left(\epsilon^{a}\right), O\left(\epsilon^{b}\right), O\left(\epsilon^{c}\right)\right),
$$

where $\epsilon(\ll 1)$ is a typical nondimensional amplitude of the pressure perturbation (or acoustic pressure) that appears in the perturbation expansions of dependent variables. The real numbers $a, b$, and $c$ in the right-hand side of Eq. (1) should be chosen appropriate to a wave concerned, for instance, Kanagawa et al. (2010) chosen so as to derive the NLS and KdVB equations, as follows:

$$
\begin{cases}a=2, b=c=0 & (\text { for NLS }) \\ a=b=c=1 / 2 & (\text { for KdVB }) .\end{cases}
$$

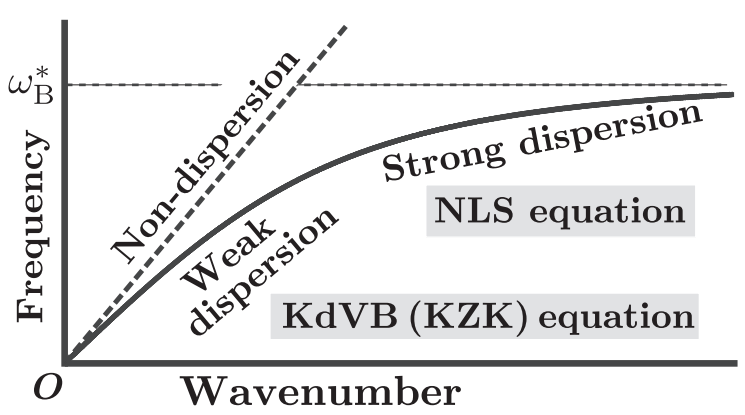

FIG. 1. Simple schematic of the linear dispersion relation of waves in an incompressible liquid containing many gas bubbles without dissipation case (van Wijngaarden, 1968, 1972). The dashed line denotes the non-dispersive waves in the absence of bubbles (i.e., acoustic waves in a single-phase liquid) and $\omega_{\mathrm{B}}^{*}$ is the eigenfrequency of single bubble oscillations.
The essence of Eq. (1) is to connect the wave amplitude with nondimensional ratios to evaluate the relative size of a ratio with respect to the nondimensional amplitude $\epsilon$. To do so, by using $\epsilon$, the right-hand side of

$$
\left(\frac{U^{*}}{c_{\mathrm{L} 0}^{*}}, \frac{\omega^{*}}{\omega_{\mathrm{B}}^{*}}, \frac{R_{0}^{*}}{L^{*}}\right) \equiv(O(A), O(B), O(C))
$$

was rewritten into that of Eq. (1) [e.g., $O\left(\epsilon^{a}\right) \equiv O(A)$ ], where $A, B$, and $C$ in the right-hand side of Eq. (3) are also the real numbers. Because three ratios in the left-hand side of Eq. (1) [or Eq. (3)] have clear physical meanings, our derivation method can offer a wider perspective for understanding complex nonlinear wave phenomena underlying bubbly liquids. The detail of procedure based on Eqs. (1)-(3) is explained in Sec. III.

The wave motions addressed in Kanagawa et al. (2010) were restricted to plane waves, i.e., one-dimensional waves in which the averaged physical quantities are functions of the time and spatial coordinate in the direction of wave propagation. Furthermore, the initial void fraction was assumed to be constant, which implies that initial number density of the bubbles in the liquid is spatially uniform. In this paper, we extend our method to encompass a sound beam in a nonuniform bubbly liquid without these restrictions. The method is extended by the following two steps (Kanagawa et al., 2011a; Yano et al., 2013):

(i) A scaling relation for the typical diameter of sound beam to the typical wavelength is added into the original set of scaling relations [Eq. (1)]. This scaling relation gives the size of the diffraction effect relative to the nonlinear effect and corresponds to a second nondimensional parameter with respect to the length scale.

(ii) The nondimensionalized initial number density of the bubbles is assumed to be nonuniform, and the nonuniformity and its axially spatial variation are assumed to be as small as the nondimensionalized wave amplitude $\epsilon$.

Using the scaling relations and the weak nonuniformity assumption, in the same manner as in Kanagawa et al. (2010), we apply the method of multiple scales (e.g., Nayfeh, 1973; Jeffrey and Kawahara, 1982) to a set of generic equations for bubbly flows. From this, we can derive two types of nonlinear wave equations: (i) the original NLS equation including dissipation, diffraction, and nonuniform terms, (ii) the original KZK equation (Zabolotskaya and Khokhlov, 1969; Kuznetsov, 1971; Hamilton, 1998) including dispersion term and nonuniform effect; hereafter we call the former the generalized NLS equation and the latter the generalized KZK equation, for simplicity. Note that the generalized KZK equation is similar to a generalized Kadomtsev-Petviashvili (KP) equation [i.e., the original KP equation (Kadomtsev and Petviashvili, 1970; Whitham, 1974) including dissipation term and nonuniform effect], and the original KZK and KP equations correspond to the original Burgers and $\mathrm{KdV}$ equations including diffraction effect, respectively.

The derivation of the generalized NLS and KZK (or KP) equations for sound beams, which incorporate weak diffraction 
and weak nonuniform effects, is the first such demonstration in the context of bubbly flow theory based on two-fluid model. Although the Davey-Stewartson equation (i.e., the twodimensional NLS equation) and the KP equation were derived (Khismatullin and Akhatov, 2001; Akhatov and Khismatullin, 2001) for uniform bubbly liquids from the mixture model (e.g., Nigmatulin, 1991), dissipation and nonuniform effects were neglected and the two-fluid model was not utilized. As in our previous derivation (Kanagawa et al., 2010, 2011a), we employ the basic equations in the two-fluid model (Egashira et al., 2004; Yano et al., 2006); the broad applicability of this model to wave propagation analysis was revealed by Kanagawa et al. (2011b). To demonstrate the wider applicability of our method, this paper tackles the derivation of two nonlinear wave equations.

This paper is organized as follows: In Sec. II, we formulate the problem. In Sec. III, we introduce the diffraction and nonuniform effects. In Sec. IV, we derive the generalized NLS equation. In Sec. V, we review the derivation of the generalized KZK equation [the detailed procedure is found in Kanagawa et al. (2011a)]. In Sec. VI, we summarize the nonlinear wave equations derived.

\section{FORMULATION OF THE PROBLEM}

\section{A. Problem statement}

The problem of nonlinear propagation of a quasi-plane (i.e., weakly diffracted) dispersive pressure wave (i.e., a sound beam) in a compressible liquid containing a large number of small spherical gas bubbles is considered. The sound beam is radiated from a circular sound source of diameter $D^{*}$ in the bubbly liquid initially at rest, and the initial distribution of the number density of the bubbles is nonuniform (the superscript asterisk denotes dimensional quantities throughout this paper). The center of the sound source defines the origin, and the central $x^{*}$ axis is adopted as the surface normal (Fig. 2). The amplitude of the sound beam is sufficiently small relative to the pressure in the ambient bubbly liquid. Our aim is to derive nonlinear wave equations governing the asymptotic behavior of the wave motions with respect to small but finite amplitude [i.e., the so-called

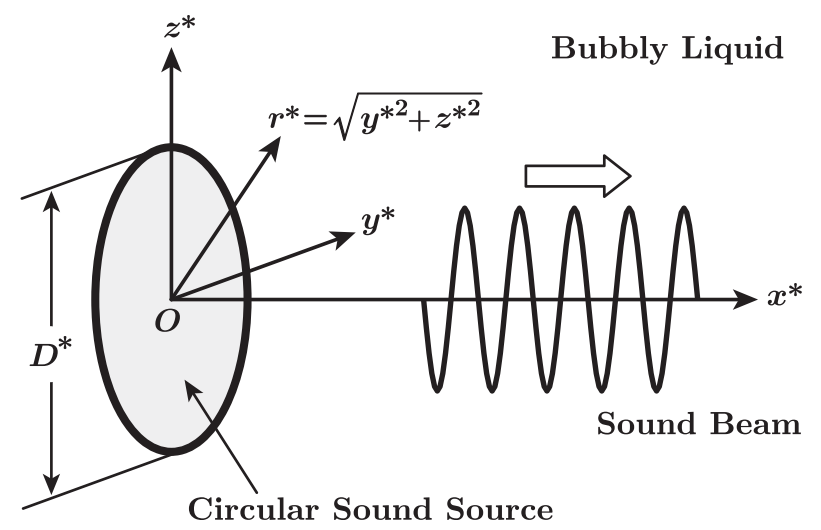

FIG. 2. Schematic of the model: nonlinear propagation of the diffractive sound beam radiated from the circular sound source in nonuniform bubbly liquids. The white arrow shows the propagating direction. weakly nonlinear problem (e.g., Nayfeh, 1973; Jeffrey and Kawahara, 1982)].

The wave motion is extended in two ways (see also Kanagawa et al., 2011a) from the plane wave problem considered by Kanagawa et al. (2010). The first extension is based on the condition that the typical wavelength $L^{*}$ is sufficiently small relative to $D^{*}$,

$$
L^{*} \ll D^{*} \text {. }
$$

As demonstrated below, this condition yields a sound beam with weak diffraction (either weak focusing or weak diffusing). The second extension is the inclusion of a nonuniform spatial distribution of the initial number density of the bubbles, $n_{0}^{*}\left(x^{*}, r^{*}\right)$. We assume that the nonuniformity is weak in the sense that

$$
\left|\frac{n_{0}^{*}}{n_{00}^{*}}-1\right| \ll 1,
$$

where $n_{00}^{*}$ is the spatial average of $n_{0}^{*}$, and is constant.

The averaged physical quantities, defined in the bubbly liquid using the volume or surface averages (Egashira et al., 2004), are assumed to be symmetric about the $x^{*}$ axis. Accordingly, all the averaged physical quantities are functions of the time, $t^{*}$, the distance from the sound source, $x^{*}$, and the distance from the $x^{*}$ axis, $r^{*}$, where

$$
r^{*}=\sqrt{y^{* 2}+z^{* 2}}
$$

\section{B. Assumptions}

To this end, we make the following assumptions: (i) The motion of a bubble is spherically symmetric. (ii) Bubbles do not appear or disappear, coalesce, and break up. (iii) The bubbly liquid is dilute such that no direct bubble-bubble interactions occur, where the method of averaged equations is effective. (iv) The flow field is quasi-irrotational, i.e., the near field is irrotational and the far field is rotational. This is justified because the wave motions considered here are quasi-planar. (v) The liquid has a small compressibility. (vi) The small viscosity of the liquid is important at only the bubble wall and the viscosity of the gas is neglected. (vii) The gas inside the bubbles is composed of only a non-condensable gas, and hence no phase change occurs across the bubble-liquid interface.

Furthermore, we also neglect the following items: (viii) bulk viscosities of the gas and liquid, (ix) thermal conductivities of the gas and liquid, and (x) forces of gravity, Reynolds stress, and other forces (e.g., drag, lift, etc.) exerted on the bubbles, apart from the virtual mass force. For (ix), although wave attenuation is caused by three effects, the liquid viscosity, liquid compressibility, and thermal conductivity (van Wijngaarden, 1972), we neglect the thermal effects for simplicity. We note, however, that thermal processes inside the bubbles with heat exchanged at the bubble-liquid interface are known to induce significant wave attenuation in bubbly liquids (e.g., Nigmatulin, 1991; Prosperetti, 1991; Watanabe and Prosperetti, 1994). For (x), forces exerted on the bubbles are generally significant (e.g., 
Drew, 1983) and should ideally not be ignored, but because the propagation of acoustic waves in an almost quiescent bubbly liquid causes small amplitude oscillations of the fluid particles, small bubbles move together with the surrounding liquid. Hence, only the virtual mass force is taken into account as a representative force because it has a strongly influence on both unsteady fluid flow and the bubbles (see also Yano et al., 2013).

\section{Basic equations}

As shown by Kanagawa et al. (2011b), the derivation method (Kanagawa et al., 2010) can be applied to both the mixture model and two-fluid model equations. Here, we employ basic equations in a two-fluid model (Egashira et al., 2004) because the use of two-fluid model gives the expression of various parameter dependences into coefficients of nonlinear wave equation (Kanagawa et al., 2011b) and the derivation of NLS and KZK equations from two-fluid model has not been performed.

The set of averaged equations for bubbly flows (Yano et al., 2006) consists of the conservation laws of mass and momentum for the gas and liquid phases based on the twofluid model (Egashira et al., 2004), equation of motion for the bubble wall, equations of state for the gas and liquid phases, and supplementary equations. For axisymmetric flows (quasi-plane waves), the conservation laws of mass and momentum for the gas and liquid phases are given by

$$
\begin{aligned}
& \frac{\partial}{\partial t^{*}}\left(\alpha \rho_{\mathrm{G}}^{*}\right)+\nabla^{*} \cdot\left(\alpha \rho_{\mathrm{G}}^{*} \boldsymbol{u}_{\mathrm{G}}^{*}\right)=0 \\
& \frac{\partial}{\partial t^{*}}\left[(1-\alpha) \rho_{\mathrm{L}}^{*}\right]+\boldsymbol{\nabla}^{*} \cdot\left[(1-\alpha) \rho_{\mathrm{L}}^{*} \boldsymbol{u}_{\mathrm{L}}^{*}\right]=0 \\
& \frac{\partial}{\partial t^{*}}\left(\alpha \rho_{\mathrm{G}}^{*} \boldsymbol{u}_{\mathrm{G}}^{*}\right)+\nabla^{*} \cdot\left(\alpha \rho_{\mathrm{G}}^{*} \boldsymbol{u}_{\mathrm{G}}^{*} \otimes \boldsymbol{u}_{\mathrm{G}}^{*}\right)+\alpha \nabla^{*} p_{\mathrm{G}}^{*}=\boldsymbol{F}^{*}, \\
& \frac{\partial}{\partial t^{*}}\left[(1-\alpha) \rho_{\mathrm{L}}^{*} \boldsymbol{u}_{\mathrm{L}}^{*}\right]+\nabla^{*} \cdot\left[(1-\alpha) \rho_{\mathrm{L}}^{*} \boldsymbol{u}_{\mathrm{L}}^{*} \otimes \boldsymbol{u}_{\mathrm{L}}^{*}\right] \\
& \quad+(1-\alpha) \nabla^{*} p_{\mathrm{L}}^{*}+P^{*} \nabla^{*} \alpha=-\boldsymbol{F}^{*}
\end{aligned}
$$

where $\alpha$ is the void fraction $(0<\alpha<1), \rho^{*}$ is the density, $\boldsymbol{u}^{*}=\left(u^{*}, v^{*}\right)$ is the fluid velocity (with $u^{*}$ and $v^{*}$ being the components in the $x^{*}$ and $r^{*}$ directions, respectively), and $p^{*}$ is the pressure, the subscripts $\mathrm{G}$ and $\mathrm{L}$ denote the volumeaveraged variables in the gas and liquid phases, respectively, and the symbol $\otimes$ denotes the tensor product. Note that $p_{\mathrm{G}}^{*}$ and $p_{\mathrm{L}}^{*}$ are the volume-averaged pressures in gas and liquid phases, respectively, and $P^{*}$ is defined as the deviation of a surface average of the local liquid pressure from the volumeaveraged liquid pressure $p_{\mathrm{L}}^{*}$ (Egashira et al., 2004), which is based on the formulation by Prosperetti and Jones (1984). Hence, the pressure acting on the interface is $p_{\mathrm{L}}^{*}+P^{*}$ (not only $\left.P^{*}\right)$.

For the interfacial momentum transport $\boldsymbol{F}^{*}$ in Eqs. (9) and (10), we employ the following model, taken from an analysis of a compressible liquid (Zhang and Prosperetti, 1994; Eames and Hunt, 2004), for the virtual mass force (Yano et al., 2006):

$$
\begin{aligned}
\boldsymbol{F}^{*}= & -\beta_{1} \alpha \rho_{\mathrm{L}}^{*}\left(\frac{\mathrm{D}_{\mathrm{G}} \boldsymbol{u}_{\mathrm{G}}^{*}}{\mathrm{D} t^{*}}-\frac{\mathrm{D}_{\mathrm{L}} \boldsymbol{u}_{\mathrm{L}}^{*}}{\mathrm{D} t^{*}}\right) \\
& -\beta_{2} \rho_{\mathrm{L}}^{*}\left(\boldsymbol{u}_{\mathrm{G}}^{*}-\boldsymbol{u}_{\mathrm{L}}^{*}\right) \frac{\mathrm{D}_{\mathrm{G}} \alpha}{\mathrm{D} t^{*}}-\beta_{3} \alpha\left(\boldsymbol{u}_{\mathrm{G}}^{*}-\boldsymbol{u}_{\mathrm{L}}^{*}\right) \frac{\mathrm{D}_{\mathrm{G}} \rho_{\mathrm{L}}^{*}}{\mathrm{D} t^{*}},
\end{aligned}
$$

where the coefficients $\beta_{1}, \beta_{2}$, and $\beta_{3}$ may be set to $1 / 2$ for a spherical bubble. We proceed without explicitly showing these values to ensure that the contribution of each term on the right-hand side of Eq. (11) is visible in the final result; $\beta_{3}$ does not appear in the final results for any of the problems studied by our group (Yano et al., 2006; Kanagawa et al., 2010, 2011a; Yano et al., 2013) including the present analysis.

The spherically symmetric oscillations of a representative bubble are described by the Keller equation:

$$
\begin{aligned}
(1 & \left.-\frac{1}{c_{\mathrm{L} 0}^{*}} \frac{\mathrm{D}_{\mathrm{G}} R^{*}}{\mathrm{D} t^{*}}\right) R^{*} \frac{\mathrm{D}_{\mathrm{G}}^{2} R^{*}}{\mathrm{D} t^{* 2}} \\
& +\frac{3}{2}\left(1-\frac{1}{3 c_{\mathrm{L} 0}^{*}} \frac{\mathrm{D}_{\mathrm{G}} R^{*}}{\mathrm{D} t^{*}}\right)\left(\frac{\mathrm{D}_{\mathrm{G}} R^{*}}{\mathrm{D} t^{*}}\right)^{2} \\
& =\left(1+\frac{1}{c_{\mathrm{L} 0}^{*}} \frac{\mathrm{D}_{\mathrm{G}} R^{*}}{\mathrm{D} t^{*}}\right) \frac{P^{*}}{\rho_{\mathrm{L} 0}^{*}}+\frac{R^{*}}{\rho_{\mathrm{L} 0}^{*} c_{\mathrm{L} 0}^{*}} \frac{\mathrm{D}_{\mathrm{G}}}{\mathrm{D} t^{*}}\left(p_{\mathrm{L}}^{*}+P^{*}\right),
\end{aligned}
$$

where $R^{*}$ is the radius of the representative bubble, $c_{\mathrm{L} 0}^{*}$ and $\rho_{\mathrm{L} 0}^{*}$ are the speed of sound and the density in the initial unperturbed liquid, respectively, and the operators $\mathrm{D}_{\mathrm{G}} / \mathrm{D} t^{*}$ and $\mathrm{D}_{\mathrm{L}} / \mathrm{D} t^{*}$ are defined as

$$
\frac{\mathrm{D}_{\mathrm{G}}}{\mathrm{D} t^{*}} \equiv \frac{\partial}{\partial t^{*}}+\boldsymbol{u}_{\mathrm{G}}^{*} \cdot \nabla^{*}, \quad \frac{\mathrm{D}_{\mathrm{L}}}{\mathrm{D} t^{*}} \equiv \frac{\partial}{\partial t^{*}}+\boldsymbol{u}_{\mathrm{L}}^{*} \cdot \nabla^{*} .
$$

The symbols in the original Keller equation (Keller and Kolodner, 1956) are replaced as follows: the liquid pressure at infinity is replaced by the averaged pressure $p_{\mathrm{L}}^{*}$, the liquid pressure at the bubble-liquid interface by $p_{\mathrm{L}}^{*}+P^{*}$, and the density and the speed of sound of the liquid at infinity by those in the initially equilibrium state. As is clear from the right-hand side in the first term of Eq. (12), $P^{*}$ corresponds to a driving force. Hence, the term $P^{*} \nabla^{*} \alpha$ in Eq. (10) expresses an interaction between the bubble oscillations driven by $P^{*}$ and bubble concentrations described by $\nabla^{*} \alpha$. The second term on the right-hand side of Eq. (12) describes the damping effect on the bubble oscillations and is mainly responsible for wave attenuation due to acoustic radiation from oscillating bubbles. The first term on the right-hand side also results in wave attenuation due to the liquid viscosity $\mu^{*}$ through Eq. (16) below.

In accordance with the assumptions in Sec. II B, since the energy conservation equations are not solved, the equations of state are then employed as supplementary equations. Note that even if thermal effects are taken into account, this will not change the essential points of our derivation methodology. The set of Eqs. (7)-(12) is closed by the following four equations: (i) the Tait equation of state for liquids, 


$$
p_{\mathrm{L}}^{*}=p_{\mathrm{L} 0}^{*}+\frac{\rho_{\mathrm{L} 0}^{*} c_{\mathrm{L} 0}^{*}}{n}\left[\left(\frac{\rho_{\mathrm{L}}^{*}}{\rho_{\mathrm{L} 0}^{*}}\right)^{n}-1\right] \text {, }
$$

where $p_{\mathrm{L} 0}^{*}$ is the liquid pressure in the initial undisturbed state and $n$ is the material constant (e.g., $n=7.15$ for water). (ii) The polytropic equation of state for gases,

$$
\frac{p_{\mathrm{G}}^{*}}{p_{\mathrm{G} 0}^{*}}=\left(\frac{\rho_{\mathrm{G}}^{*}}{\rho_{\mathrm{G} 0}^{*}}\right)^{\gamma}
$$

where $p_{\mathrm{G} 0}^{*}$ and $\rho_{\mathrm{G} 0}^{*}$ are the pressure and the density inside the bubble in the initial state, respectively, and $\gamma$ is the polytropic exponent. (iii) The conservation law of mass inside the bubble,

$$
\frac{p_{\mathrm{G}}^{*}}{p_{\mathrm{G} 0}^{*}}=\left(\frac{R_{0}^{*}}{R^{*}}\right)^{3},
$$

where $R_{0}^{*}$ is the bubble radius in the initial state. (iv) The balance of normal stresses across the bubble-liquid interface,

$$
p_{\mathrm{G}}^{*}-\left(p_{\mathrm{L}}^{*}+P^{*}\right)=\frac{2 \sigma^{*}}{R^{*}}+\frac{4 \mu^{*}}{R^{*}} \frac{\mathrm{D}_{\mathrm{G}} R^{*}}{\mathrm{D} t^{*}},
$$

where $\sigma^{*}$ is the surface tension. The effect of the liquid viscosity $\mu^{*}$ is considered only at the bubble-liquid interface, as noted in Sec. II B (vi). The physical quantities in the initial undisturbed state, which are signified by the subscript 0, are all constants, except for $n_{0}^{*}$ in Eq. (5) [or Eq. (40)].

\section{INCORPORATION OF DIFFRACTION AND NONUNIFORMITY}

\section{A. Parameter scaling}

As shown in Eqs. (1)-(3) in Sec. I, we here introduce the scaling relations among the physical parameters and explain the procedure to determine the parameter scaling (Kanagawa et al., 2010; Yano et al., 2013) for the derivation of generalized NLS and KZK equations.

The first target is a high-frequency, large-wavenumber, weakly nonlinear wave in a bubbly liquid. The basic properties of this wave are determined by the linear dispersion relation. As shown in Fig. 1, the difference between the dashed line (the nondispersive wave) and the solid curve is an increasing function of the wavenumber. Hence, waves with large wavenumber are identified as being strongly dispersive, i.e., a typical propagation speed of wave, $U^{*}$, is much smaller than the speed of sound in the absence of bubbles, $c_{\mathrm{L} 0}^{*}$. The physical relation between $U^{*}$ and $c_{\mathrm{L} 0}^{*}$ is thus $U^{*} /$ $c_{\mathrm{L} 0}^{*} \ll 1$; the right-hand side should be expressed by using a parameter that estimates the order of $U^{*} / c_{\mathrm{L} 0}^{*}$. We then introduce the nondimensional parameter (perturbation), $\epsilon(\ll 1)$, which is also introduced into variables in Secs. IIIC and IIID. We rewrite the inequality above as

$$
\frac{U^{*}}{c_{\mathrm{L} 0}^{*}} \equiv O(A) \equiv O\left(\epsilon^{a}\right) \equiv O\left(\epsilon^{2}\right) \equiv V \epsilon^{2}
$$

where $A$ and $a$ determine the order [see also Eqs. (1)-(3) in Sec. I], and $V$ is a constant of $O(1)$. The procedure of order determination in Eq. (17a) is as follows: (i) The dependence of $A$ on the nonlinearity $\epsilon$ is assumed to evaluate the relative size of $U^{*} / c_{\mathrm{L} 0}^{*}$ to $\epsilon$. (ii) The real number $a$ is then introduced for an order estimation. (iii) For example, in the derivation of the generalized NLS equation, the choice of $a=2$ is the uniqueness (another choice derives another nonlinear wave equation); in other words, this is an inverse calculation.

We are focusing on the generalized NLS equation because it describes a simple balance as the linear combination of the dissipation, dispersion, diffraction, nonlinearity, and nonuniformity, such as the Burgers or KdV equations for single-phase fluids. Generally speaking, the set of nondimensional ratios in the left-hand side of Eq. (1) should be treated dependently on various parameters. Nevertheless, the perturbation analysis with respect to the nondimensional amplitude $\epsilon$ yields the systematic derivation of various weakly nonlinear waves in the $\epsilon$ dependence, as useful information laying in the complex set of basic equations (7)-(10). Therefore, we assume that all the nondimensional ratios [including Eqs. (17b), (17c), etc.] depend on $\epsilon$, and this treatment is our essence.

By using the same procedure in Eq. (17a) with Fig. 1 because a typical angular frequency of the sound source (wave frequency), $\omega^{*}$, is assumed to be comparable to the eigenfrequency of the bubble, $\omega_{\mathrm{B}}^{*}$, and the wavelength, $L^{*}$, is comparable to the typical radius of a representative bubble, $R_{0}^{*}$, we can write down

$$
\begin{aligned}
& \frac{\omega^{*}}{\omega_{\mathrm{B}}^{*}} \equiv T^{*} \omega^{*} \equiv O(1) \equiv \Omega, \\
& \frac{R_{0}^{*}}{L^{*}} \equiv O(1) \equiv \Delta,
\end{aligned}
$$

where $\Omega$ and $\Delta$ are also constants of $O(1) ; \Omega$ is the normalized angular frequency. The validity of Eq. (17) is discussed in the context of the derivation of the NLS equation (Sec. IV).

As the second target, for a low-frequency small-wavenumber wave, $U^{*}$ is small compared to $c_{\mathrm{L} 0}^{*}$ (Fig. 1). We define such a wave as

$$
\frac{U^{*}}{c_{\mathrm{L} 0}^{*}} \equiv O(\sqrt{\epsilon}) \equiv V \sqrt{\epsilon}
$$

Furthermore, $\omega^{*}$ and $R_{0}^{*}$ are assumed to be small compared to $\omega_{\mathrm{B}}^{*}$ and $L^{*}$, respectively, which can be expressed as follows:

$$
\begin{aligned}
& \frac{\omega^{*}}{\omega_{\mathrm{B}}^{*}} \equiv \frac{1}{T^{*} \omega_{\mathrm{B}}^{*}} \equiv O(\sqrt{\epsilon}) \equiv \Omega \sqrt{\epsilon}, \\
& \frac{R_{0}^{*}}{L^{*}} \equiv O(\sqrt{\epsilon}) \equiv \Delta \sqrt{\epsilon}
\end{aligned}
$$


In Eqs. (17) and (18), $L^{*}=U^{*} T^{*}$ is assumed and the characteristic times $T^{*}$ in Eqs. (17b) and (18b) are expressed as

$$
T^{*} \equiv \begin{cases}1 / \omega_{\mathrm{B}}^{*} & (\text { for NLS }) \\ 1 / \omega^{*} & (\text { for KZK })\end{cases}
$$

The two scaling relations in Eqs. (17) and (18) are combined into a set of nondimensional parameters:

$$
\left(\frac{U^{*}}{c_{\mathrm{L} 0}^{*}}, \frac{\omega^{*}}{\omega_{\mathrm{B}}^{*}}, \frac{R_{0}^{*}}{L^{*}}\right) \equiv \begin{cases}\left(O\left(\epsilon^{2}\right), O(1), O(1)\right) & (\text { for NLS }), \\ (\mathrm{O}(\sqrt{\epsilon}), O(\sqrt{\epsilon}), O(\sqrt{\epsilon})) & (\text { for KZK }) .\end{cases}
$$

Incidentally, the common method for averaged equations (7)-(10) is usually prohibited for the short waves, i.e., $R_{0}^{*} \simeq L^{*}$ in Eq. (17c); however, the quasi-plane wave assumption can be applied because the average volume is sufficiently large along the plane parallel to the quasi-plane wavefronts. Nevertheless, it is worth noting that the assumption of spherically symmetric bubble oscillations (Egashira et al., 2004) must be validated.

The eigenfrequency of the linear spherically symmetric oscillations of a single bubble is given by

$$
\frac{\omega_{\mathrm{B}}^{*}}{2 \pi} \equiv \frac{1}{2 \pi} \sqrt{\frac{3 \gamma\left(p_{\mathrm{L} 0}^{*}+2 \sigma^{*} / R_{0}^{*}\right)-2 \sigma^{*} / R_{0}^{*}}{\rho_{\mathrm{L} 0}^{*} R_{0}^{* 2}}} .
$$

Note that the effects of liquid viscosity and liquid compressibility are not included in Eq. (21).

The magnitude of the normalized liquid viscosity is also assumed to be small compared to unity, so that the dissipation effect caused by the liquid viscosity competes with the dissipation effect caused by the liquid compressibility and with the nonlinear, dispersion, diffraction, and nonuniform effects, which is a result of the long-range propagation of the sound beams. The normalized liquid viscosity $\mu$ is thus defined as

$$
\frac{\mu^{*}}{\rho_{\mathrm{L} 0}^{*} U^{*} L^{*}} \equiv \begin{cases}O\left(\epsilon^{2}\right) \equiv \mu \epsilon^{2} & (\text { for NLS }), \\ O(\epsilon) \equiv \mu \epsilon & (\text { for KZK }) .\end{cases}
$$

The ratio of the initial densities of the gas and liquid phases is also assumed to be small,

$$
\frac{\rho_{\mathrm{G} 0}^{*}}{\rho_{\mathrm{L} 0}^{*}} \equiv O\left(\epsilon^{3}\right) \quad(\text { for NLS and KZK }),
$$

and hence, the density ratio does not affect the final result of the present analysis.

\section{B. Diffraction effect}

We define the large aperture condition in Eq. (4), $L^{*} \ll D^{*}$, as a function of $\epsilon$,

$$
\frac{L^{*}}{D^{*}} \equiv \begin{cases}O(\epsilon) \equiv \Gamma \epsilon & (\text { for NLS }) \\ O(\sqrt{\epsilon}) \equiv \Gamma \sqrt{\epsilon} & (\text { for KZK })\end{cases}
$$

where $\Gamma$ is a constant of $O(1)$ and represents the effect of weak diffraction to the $r$ direction. The magnitude of $L^{*} / D^{*}$ characterizes the pattern of the sound beams (e.g., Zemanek, 1971; Blackstock, 2000). The scaling $L^{*} / D^{*} \ll 1$ ensures that the sound beam is approximately unidirectional and localized in the vicinity of the $x^{*}$ axis. Thus, the wavefronts are quasi-planar (Hamilton, 1998).

Equations (24) [or (4)], (17c), and (18c) summarize the relation among the three length scales as

$$
\begin{cases}R_{0}^{*} \simeq L^{*} \ll D^{*} & (\text { for } \mathrm{NLS}), \\ R_{0}^{*} \ll L^{*} \ll D^{*} & (\text { for } \mathrm{KZK}) .\end{cases}
$$

\section{Multiple-scale expansions}

The nondimensional independent variables $t, x$, and $r$ are defined as

$$
t=\frac{t^{*}}{T^{*}}, \quad x=\frac{x^{*}}{L^{*}}, \quad r=\frac{r^{*}}{L^{*}} .
$$

For weakly nonlinear problems, the nonlinear effect manifests itself in the far field (i.e., $x^{*} \gg L^{*}$; the near field is defined as the region close to the sound source). As the problem involves different time and length scales, the method of multiple scales (e.g., Nayfeh, 1973; Jeffrey and Kawahara, 1982) is generally effective for such problems. In this method, various phenomena are described via extended independent-variables. Because the diffraction effect does not appear up to the far field, the spatial variation of the beam in the $r$ direction is small compared to that in the $x$ direction. The extended independent-variables are

$$
\left\{\begin{array}{lll}
t_{m}=\epsilon^{m} t, & x_{m}=\epsilon^{m} x(m=0,1,2), \quad r_{1}=\epsilon \Gamma r & (\text { for NLS }), \\
t_{m}=\epsilon^{m} t, & x_{m}=\epsilon^{m} x(m=0,1), & r_{1 / 2}=\sqrt{\epsilon} \Gamma r
\end{array} \quad\right. \text { (for KZK). }
$$

For the near field characterized by $t_{0}(=t)$ and $x_{0}(=x)$ for the generalized NLS and KZK equations, and the far field characterized by $t_{1}(=\epsilon t)$ and $x_{1}(=\epsilon x)$ for the generalized NLS equation (hereafter referred to as Far-field I for only the NLS case), the wavefront of the sound beam is approximately planar (Hamilton, 1998). Because of this, we can ignore the spatial variations in the $r$ direction of the dependent variables and employ the independent-variables defined by only $t$ and $x$,

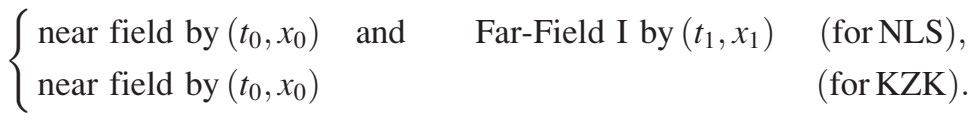


On the other hand, if the far field is characterized by spherical wavefronts (hereafter referred to as Far-field II for only the NLS case), the radial dependence of the dependent variables should be taken into account (Hamilton, 1998), and hence, the description of the wave motion will be a function of both $x$ and $r$. The nonlinear and diffraction effects of the sound beam that appear in both far fields can be characterized by

$$
\begin{cases}\text { Far-field II by }\left(t_{2}, x_{2}, r_{1}\right) & (\text { for NLS), } \\ \text { far field by }\left(t_{1}, x_{1}, r_{1 / 2}\right) & \text { (for KZK). }\end{cases}
$$

The fast scales $\left(x_{1}, r_{1 / 2}\right)$ and $\left(x_{2}, r_{1}\right)$ imply that spatial variations in the $r$ axis are slower than those in the $x$ axis. The definitions of $r_{1}$ and $r_{1 / 2}$ in Eq. (27) are consistent with the scaling relation in Eq. (24):

$$
r=\frac{D^{*}}{L^{*}} \frac{r^{*}}{D^{*}}= \begin{cases}\frac{r_{1}}{\epsilon \Gamma}\left(r_{1} \equiv \frac{r^{*}}{D^{*}}\right) & (\text { for NLS }), \\ \frac{r_{1 / 2}}{\sqrt{\epsilon} \Gamma}\left(r_{1 / 2} \equiv \frac{r^{*}}{D^{*}}\right) & (\text { for KZK). }\end{cases}
$$

All the dependent variables can now be regarded as functions of the extended independent-variables in Eqs. (28) and (29). The differential operators are thus expanded as

$$
\begin{aligned}
& \frac{\partial}{\partial t}= \begin{cases}\frac{\partial}{\partial t_{0}}+\epsilon \frac{\partial}{\partial t_{1}}+\epsilon^{2} \frac{\partial}{\partial t_{2}} & \text { (for NLS) } \\
\frac{\partial}{\partial t_{0}}+\epsilon \frac{\partial}{\partial t_{1}} & \text { (for KZK) },\end{cases} \\
& \frac{\partial}{\partial x}= \begin{cases}\frac{\partial}{\partial x_{0}}+\epsilon \frac{\partial}{\partial x_{1}}+\epsilon^{2} \frac{\partial}{\partial x_{2}} & \text { (for NLS), } \\
\frac{\partial}{\partial x_{0}}+\epsilon \frac{\partial}{\partial x_{1}} & \text { (for KZK), }\end{cases}
\end{aligned}
$$

$$
\frac{\partial}{\partial r}= \begin{cases}\epsilon \Gamma \frac{\partial}{\partial r_{1}} & (\text { for NLS }) \\ \sqrt{\epsilon} \Gamma \frac{\partial}{\partial r_{1 / 2}} & (\text { for KZK }) .\end{cases}
$$

The dependent variables are also expanded as a power series in $\epsilon(\ll 1)$. The expansions of the fluid velocities are

$$
\begin{aligned}
& u_{\mathrm{G}}^{*} / U^{*}=\epsilon u_{\mathrm{G} 1}+\epsilon^{2} u_{\mathrm{G} 2}+\cdots, \\
& u_{\mathrm{L}}^{*} / U^{*}=\epsilon u_{\mathrm{L} 1}+\epsilon^{2} u_{\mathrm{L} 2}+\cdots, \\
& v_{\mathrm{G}}^{*} / U^{*}= \begin{cases}\epsilon^{2} v_{\mathrm{G} 1}+\epsilon^{3} v_{\mathrm{G} 2}+\cdots & \text { (for NLS), } \\
\epsilon^{3 / 2} v_{\mathrm{G} 1}+\epsilon^{5 / 2} v_{\mathrm{G} 2}+\cdots & \text { (for KZK), }\end{cases} \\
& v_{\mathrm{L}}^{*} / U^{*}= \begin{cases}\epsilon^{2} v_{\mathrm{L} 1}+\epsilon^{3} v_{\mathrm{L} 2}+\cdots & \text { (for NLS), } \\
\epsilon^{3 / 2} v_{\mathrm{L} 1}+\epsilon^{5 / 2} v_{\mathrm{L} 2}+\cdots & \text { (for KZK) } .\end{cases}
\end{aligned}
$$

Other dependent variables, the bubble radius $R^{*}$, liquid density $\rho_{\mathrm{L}}^{*}$, and liquid pressure $p_{\mathrm{L}}^{*}$, are expanded as in Kanagawa et al. (2010):

$$
\begin{aligned}
& R^{*} / R_{0}^{*}-1=\epsilon R_{1}+\epsilon^{2} R_{2}+\cdots, \\
& \rho_{\mathrm{L}}^{*} / \rho_{\mathrm{L} 0}^{*}-1= \begin{cases}\epsilon^{5} \rho_{\mathrm{L} 1}+\epsilon^{6} \rho_{\mathrm{L} 2}+\cdots & (\text { for NLS }), \\
\epsilon^{2} \rho_{\mathrm{L} 1}+\epsilon^{3} \rho_{\mathrm{L} 2}+\cdots & (\text { for KZK }),\end{cases} \\
& \frac{p_{\mathrm{L}}^{*}-p_{\mathrm{L} 0}^{*}}{\rho_{\mathrm{L} 0}^{*} U^{* 2}}=\epsilon p_{\mathrm{L} 1}+\epsilon^{2} p_{\mathrm{L} 2}+\cdots,
\end{aligned}
$$

In this way, the perturbations of the dependent variables (including the void fraction $\alpha$ ) take the form of asymptotic expansions with the first term being of the order of $\epsilon$. It is worth noting that the following exceptions:

(i) The magnitude of the variations in the radial ( $r$ directional) velocities, $v_{\mathrm{G}}^{*}$ and $v_{\mathrm{L}}^{*}$, are smaller than those in the axial ( $x$ directional) velocities, $u_{\mathrm{G}}^{*}$ and $u_{\mathrm{L}}^{*}$ [Eqs. (34) and (35)]. The first terms of the expansions of radial velocities begin with $O\left(\epsilon^{2}\right)$ and $O\left(\epsilon^{3 / 2}\right)$ in the derivation of the generalized NLS and KZK equations, respectively, because of the definition of $r_{1}$ and $r_{1 / 2}$ in Eq. (27). We emphasize that these expansions based on parameter scaling are unique choices so as to derive the generalized NLS and KZK equations that describe one of the most simple balances of various wave properties.

(ii) The first terms of the expansion of the liquid density $\rho_{\mathrm{L}}^{*}[\mathrm{Eq}$. (37)] are higher-order terms, because the propagation speeds of two sound beams considered are smaller than the speed of sound in the absence of bubbles. Detailed explanation of the treatment of the variation of $\rho_{\mathrm{L}}^{*}$ [i.e., the size of the liquid compressibility described by $U^{*} / c_{\mathrm{L} 0}^{*}$ in Eqs. (17a) and (18a)] was given by Kanagawa et al. (2010). 


\section{Nonuniform bubble number density}

The expansion of the bubble number density is equivalent to that of the void fraction that is expanded in the form

$$
\alpha / \alpha_{0}-1= \begin{cases}\epsilon \alpha_{1}+\epsilon^{2}\left[\alpha_{2}+\delta\left(x_{2}\right)\right]+\epsilon^{3} \alpha_{3}+\cdots & \text { (for NLS), } \\ \epsilon\left[\alpha_{1}+\delta\left(x_{1}\right)\right]+\epsilon^{2} \alpha_{2}+\epsilon^{3} \alpha_{3}+\cdots & \text { (for KZK), }\end{cases}
$$

where $\delta$ denotes the nonuniformity of the initial-state bubble distribution. In the derivation of the generalized NLS and KZK equations, because the sound beam spreads out only up to a range of $O(1 / \epsilon)$ and $O(1 / \sqrt{\epsilon})$ in the $r$ direction, $\delta$ is regarded as a function of $x_{2}$ and $x_{1}$, respectively. The initial void fraction $\alpha_{0}$ is of the order of unity and is constant. As the bubble oscillations are assumed to be spherically symmetric and strong interactions among the bubbles are ignored, this requires that the condition $\alpha_{0} \ll 1$ is satisfied. However, we can define $\alpha_{0}=O(1)$ because we are performing an asymptotic analysis for $\epsilon \ll 1$ [Eqs. (32)-(39)]. Hence, the present analysis can also be applied to a moderately large $\alpha_{0}$.

The initial nonuniformities of the void fractions, $\delta\left(x_{1}\right)$ and $\delta\left(x_{2}\right)$, are related to the number density of the bubbles, $n^{*}$, by the following relation, as in Eq. (5):

$$
\begin{cases}\frac{n_{0}^{*}\left(x_{2}\right)}{n_{00}^{*}}-1=\epsilon^{2} \delta\left(x_{2}\right) \ll 1 & (\text { for NLS }), \\ \frac{n_{0}^{*}\left(x_{1}\right)}{n_{00}^{*}}-1=\epsilon \delta\left(x_{1}\right) \ll 1 & (\text { for KZK }),\end{cases}
$$

through the definition of the void fraction

$$
\alpha \equiv \frac{4 \pi}{3} R^{* 3} n^{*} .
$$

Before proceeding with the derivation of the generalized NLS and KZK equations, note that the same symbols (e.g., $U^{*}, \delta$, etc.) are sometimes employed in both cases without re-definition.

\section{GENERALIZED NLS EQUATION}

In this section, we consider the propagation of shortwavelength high-frequency sound beams, and derive the generalized NLS equation as a far field equation in which the diffraction and nonuniform effects compete with the nonlinear effect caused by long-range propagation.

\section{A. Linear propagation of envelope}

We substitute the expansions [Eqs. (31)-(39)] and the scaling relation [Eqs. (17) and (24)] for the generalized NLS equation into the basic set [Eqs. (7)-(16)] and equate the coefficients of like powers of $\epsilon$ to obtain a set of first-order linear equations [as was shown explicitly in Eqs. (60)-(64) in Sec. 4.1 of Kanagawa et al. (2010)]. By eliminating the first-order perturbations except for the bubble radius $R_{1}$ from the linear set, we obtain a single linear wave equation for $R_{1}$ including a dispersion term:

$$
\begin{aligned}
\mathcal{L}_{1}\left[R_{1}\right]= & \frac{\partial^{2} R_{1}}{\partial t_{0}^{2}}-\left[\frac{\Delta^{2}}{3 \alpha_{0}}+\frac{\left(1-\alpha_{0}+\beta_{1}\right) \gamma p_{\mathrm{G} 0}}{\beta_{1}\left(1-\alpha_{0}\right)}\right] \\
& \times \frac{\partial^{2} R_{1}}{\partial x_{0}^{2}}-\frac{\Delta^{2}}{3 \alpha_{0}} \frac{\partial^{4} R_{1}}{\partial x_{0}^{2} \partial t_{0}^{2}}=0 .
\end{aligned}
$$

A single inhomogeneous equation for $R_{2}$ is established as the counterpart of Eq. (42) using the same procedure after deriving the counterparts in $O\left(\epsilon^{2}\right)$ as

$$
\mathcal{L}_{1}\left[R_{2}\right]=\Psi A^{2} e^{2 i \theta}+i\left(-\frac{\partial D}{\partial \Omega}\right)\left(\frac{\partial A}{\partial t_{1}}+v_{\mathrm{g}} \frac{\partial A}{\partial x_{1}}\right) e^{i \theta}+\text { c.c. }
$$

where $D(k, \Omega)=0$ is the linear dispersion relation that gives the group velocity $v_{\mathrm{g}}$ [see Eqs. (70) and (73) in Sec. 4.1 of Kanagawa et al. (2010)], and the nonlinear coefficient $\Psi$ is a real constant [see Eqs. (A.3) and (A.4) in Appendix 2 of Kanagawa et al. (2010)] where $\Gamma$ is used in place of $\Psi$ in this paper. Note that $i$ denotes the imaginary unit, c.c. the complex conjugate, and $A$ and $\theta$ appear in Eq. (44) below.

For the first-order equation (42), owing to the dispersion effect in the third term on the right-hand side, if an initial wave is a superposition of different harmonic components, the wave profile is separated to distinct components with their own propagation speeds. We thus assumed the solution of Eq. (42) takes the form of a quasi-monochromatic wave train that evolves into a slowly modulated wave packet (e.g., Jeffrey and Kawahara, 1982):

$$
\begin{aligned}
& R_{1}=A\left(t_{1}, t_{2} ; x_{1}, x_{2} ; r_{1}\right) e^{i \theta}+\text { c.c. }, \\
& \theta=k x_{0}-\Omega(k) t_{0}
\end{aligned}
$$

where $A$ is a slowly varying complex amplitude depending on only slow scales. The normalized frequency is $\Omega$, and normalized wavenumber is $k \equiv k^{*} L^{*}=k^{*} U^{*} / \omega_{\mathrm{B}}^{*}$ with $k^{*}$ being the wavenumber. Here, $e^{i \theta}$ and $A$ correspond to the high-frequency carrier wave and envelope wave, respectively (e.g., Jeffrey and Kawahara, 1982). Equation (44b) implies that we only focus on the right-running carrier wave. Equation (44) also indicates that the linear solution (linear approximation) leads to a frequency dependent function. The derivative of the group velocity $v_{\mathrm{g}}$ with respect to $k$, which describes the dispersion effect, is

$$
q \equiv \frac{\mathrm{d} v_{\mathrm{g}}}{\mathrm{d} k}=\frac{\mathrm{d}^{2} \Omega}{\mathrm{d} k^{2}}=-\frac{9 \alpha_{0} \Delta^{2} \Omega}{\left(3 \alpha_{0}+\Delta^{2} k^{2}\right)^{2}} \leqq 0 .
$$

The typical propagation speed $U^{*}$ in Eq. (17a) is determined so that the normalized phase velocity $v_{\mathrm{p}} \equiv \Omega / k$ is equal to unity when $\Omega=1$ (i.e., $k=1$ ). This is satisfied by choosing

$$
U^{*} \equiv \sqrt{\frac{\left(1-\alpha_{0}+\beta_{1}\right) \gamma\left(p_{\mathrm{L} 0}^{*}+2 \sigma^{*} / R_{0}^{*}\right)}{\beta_{1}\left(1-\alpha_{0}\right) \rho_{\mathrm{L} 0}^{*}}},
$$

which simultaneously determines $L^{*} \equiv U^{*} T^{*}$ through $T^{*}=1 / \omega_{\mathrm{B}}^{*}$. 
For the second-order equation (43), from the solvability condition that is equivalent to the non-secular condition of the asymptotic expansions in Eqs. (32)-(39), the coefficient of $e^{i \theta}$ on the right-hand side vanishes (e.g., Jeffrey and Kawahara, 1982), resulting in

$$
\frac{\partial A}{\partial t_{1}}+v_{\mathrm{g}} \frac{\partial A}{\partial x_{1}}=0
$$

The complex amplitude $A$ is constant in the near field characterized by $t_{0}$ and $x_{0}$, but in Far-field I by $t_{1}$ and $x_{1}$, it is only constant along the characteristic curve $\mathrm{d} x_{1} / \mathrm{d} t_{1}=v_{\mathrm{g}}$. This implies that the wave train is slowly varying. The variations in the radial direction do not appear at this stage, and the nonlinear, dissipation, and diffraction effects of $A$ and the nonuniform effect of the bubble distribution appear in Far-field II characterized by $t_{2}, x_{2}$, and $r_{1}$.

\section{B. Radial velocity and variation}

We examine the radial variations in the fluid velocities that produce a diffraction effect.

The conservation equations of the momentum in the $r$ direction for the gas and liquid phases from the approximation of $O\left(\epsilon^{2}\right)$ are

$$
\begin{aligned}
& \beta_{1} \frac{\partial v_{\mathrm{G} 1}}{\partial t_{0}}-\beta_{1} \frac{\partial v_{\mathrm{L} 1}}{\partial t_{0}}=3 \Gamma \gamma p_{\mathrm{G} 0} \frac{\partial R_{1}}{\partial r_{1}}, \\
& \left(1-\alpha_{0}+\beta_{1} \alpha_{0}\right) \frac{\partial v_{\mathrm{L} 1}}{\partial t_{0}}-\beta_{1} \alpha_{0} \frac{\partial v_{\mathrm{G} 1}}{\partial t_{0}}=-\Gamma\left(1-\alpha_{0}\right) \frac{\partial p_{\mathrm{L} 1}}{\partial r_{1}},
\end{aligned}
$$

which involve time derivatives of $v_{\mathrm{G} 1}$ and $v_{\mathrm{L} 1}$, and spatial derivatives with respect to $r_{1}$. That is, in the approximations of $O\left(\epsilon^{2}\right)$, the first-order variations of the fluid velocities in the $r$ direction and variations to the $r$ direction appear. A modification of Eq. (48) provides

$$
\frac{\partial v_{\mathrm{G} 1}}{\partial t_{0}}=\Gamma D_{1} \frac{\partial R_{1}}{\partial r_{1}}, \quad \frac{\partial v_{\mathrm{L} 1}}{\partial t_{0}}=\Gamma D_{2} \frac{\partial R_{1}}{\partial r_{1}},
$$

with

$$
\begin{aligned}
& D_{1}=\frac{3 \gamma p_{\mathrm{G} 0}\left(1-\alpha_{0}+\beta_{1} \alpha_{0}\right)}{\beta_{1}\left(1-\alpha_{0}\right)}-\Delta^{2}\left(\Omega^{2}-1\right), \\
& D_{2}=\frac{3 \alpha_{0} \gamma p_{\mathrm{G} 0}}{1-\alpha_{0}}-\Delta^{2}\left(\Omega^{2}-1\right),
\end{aligned}
$$

which defines $v_{\mathrm{G} 1}$ and $v_{\mathrm{L} 1}$ as functions of $t_{0}, x_{0}$, and $r_{1}$. Accordingly, Eq. (49) governs the wave motion on a time scale of $T^{*}$, a length scale of $L^{*}$ in the axial direction, and a length scale of $D^{*}$ in the radial direction.

\section{Nonlinear propagation of diffracted envelope with nonuniformity}

Diffraction and nonuniform effects appear in Far-field II, which is of a significant distance from Far-field I, and the generalized NLS equation derived here for the nonlinear modulation of sound beam envelopes in high-frequency carrier waves will reflect the competition among the weak dissipation, weak diffraction, weak nonuniform, and strong dispersion effects.

From the approximation of $O\left(\epsilon^{3}\right)$, the single equation for $R_{3}$ is firstly obtained:

$$
\begin{aligned}
\mathcal{L}_{1}\left[R_{3}\right]= & \tilde{N}\left(x_{0}, x_{1}, x_{2} ; t_{0}, t_{1}, t_{2} ; r_{1}\right) \\
= & -\frac{1}{3} \frac{\partial \tilde{N}_{1}}{\partial t_{0}}+\frac{1}{3 \alpha_{0}} \frac{\partial \tilde{N}_{2}}{\partial t_{0}}+\frac{1-\alpha_{0}+\beta_{1}}{3 \beta_{1}\left(1-\alpha_{0}\right)} \frac{\partial \tilde{N}_{3}}{\partial x_{0}} \\
& +\frac{1}{3 \alpha_{0}\left(1-\alpha_{0}\right)} \frac{\partial \tilde{N}_{4}}{\partial x_{0}}-\frac{\Delta^{2}}{3 \alpha_{0}} \frac{\partial^{2} \tilde{N}_{5}}{\partial x_{0}^{2}},
\end{aligned}
$$

where $\tilde{N}_{i}(i=1,2,3,4,5)$ are the inhomogeneous terms which are composed of the first- and second-order perturbations derived in the calculations of $O(\epsilon)$ and $O\left(\epsilon^{2}\right)$; the explicit forms are shown in the Appendix. Secondly, the conservation equations of the momentum in the $r$ direction for the gas and liquid phases of $O\left(\epsilon^{3}\right)$ reduce to

$$
\begin{aligned}
& \beta_{1} \frac{\partial v_{\mathrm{G} 2}}{\partial t_{0}}-\beta_{1} \frac{\partial v_{\mathrm{L} 2}}{\partial t_{0}}=S_{1}, \\
& \left(1-\alpha_{0}+\beta_{1} \alpha_{0}\right) \frac{\partial v_{\mathrm{L} 2}}{\partial t_{0}}-\beta_{1} \alpha_{0} \frac{\partial v_{\mathrm{G} 2}}{\partial t_{0}}=S_{2},
\end{aligned}
$$

where the explicit forms of $S_{1}$ and $S_{2}$ are also given in Appendix. The modification of the inhomogeneous term in Eq. (51) yields

$$
\mathcal{L}_{1}\left[R_{3}\right]=\tilde{\Lambda}_{1} e^{3 i \theta}+\tilde{\Lambda}_{2} e^{2 i \theta}+\tilde{\Lambda}_{3} e^{i \theta}+\text { c.c. },
$$

where $\tilde{\Lambda}_{i}(i=1,2,3)$ are the complex variables composed of $A$ and its derivatives. Since the explicit forms of $\tilde{\Lambda}_{1}$ and $\tilde{\Lambda}_{2}$, and those of $v_{\mathrm{G} 2}$ and $v_{\mathrm{L} 2}$ calculated from Eq. (52), are not essential for the following discussion, they are omitted here for conciseness.

Imposing the non-secular condition on Eq. (51), i.e., $\tilde{\Lambda}_{3}=0$, gives

$$
\begin{aligned}
& i\left(\frac{\partial A}{\partial t_{2}}+v_{\mathrm{g}} \frac{\partial A}{\partial x_{2}}\right)+\frac{q}{2} \frac{\partial^{2} A}{\partial x_{1}^{2}}+\nu_{1}|A|^{2} A \\
& \quad+\left(i \nu_{2}+\delta \nu_{4}\right) A+\frac{\Gamma^{2} v_{\mathrm{g}}}{2 k} \frac{1}{r_{1}} \frac{\partial}{\partial r_{1}}\left(r_{1} \frac{\partial A}{\partial r_{1}}\right)=0
\end{aligned}
$$

where the nonlinear coefficient $\nu_{1}(\leq 0)$ (real) is shown in Eq. (A.5) in Appendix 2 of Kanagawa et al. (2010). The dissipation coefficient $\nu_{2}$ (real) is given as

$$
\nu_{2}(k)=\frac{\left(4 \mu+\Delta^{3} V\right) k^{2}}{2\left(3 \alpha_{0}+\Delta^{2} k^{2}\right)} \geq 0
$$

and the dispersion coefficient $q / 2(\leq 0)$ (real) was given in Eq. (45).

The size of the bubble nonuniformity is governed by the real constant $\nu_{4}$ (nonuniform coefficient) given by

$$
\nu_{4}=\frac{\left[\beta_{1}+\left(1-\alpha_{0}\right)^{2}\right] \Delta^{2} k^{2}\left(1-\Omega^{2}\right)-3 \beta_{1} \alpha_{0}^{2} \Omega^{2}}{2\left(1-\alpha_{0}\right)\left(1-\alpha_{0}+\beta_{1}\right)\left(\Delta^{2} k^{2}+3 \alpha_{0}\right) \Omega} .
$$


Figure 3 depicts the dependence of $\nu_{4}$ on the wavenumber $k$ for the initial bubble radius of $R_{0}^{*}=1 \mathrm{~mm}$ and initial void fractions of $\alpha_{0}=10^{-1}, 10^{-2}$, and $10^{-3}$. Clearly, $\nu_{4}$ is dependent on the initial void fraction. By decomposing $\Delta$ as

$$
\Delta=\frac{R_{0}^{*}}{L^{*}}=\frac{R_{0}^{*} \omega_{\mathrm{B}}^{*}}{U^{*}},
$$

we can confirm that $\nu_{4}$ is negligibly dependent on the initial bubble radius $R_{0}$ [see Eq. (21)]. Here, $\nu_{4}$ is positive for $\Omega \lessgtr 1$, negative for $\Omega \geq 1$, and equal to zero for $\Omega<1$ for an air-water system under the ambient conditions given in the caption of Fig. 3. For the case of $\Omega=1$, we have $\nu_{4} \approx 0$, which is a slightly negative value. As can be seen from the dispersion relation in Fig. 1, however, the condition $\Omega \geq 1$ (i.e., $\omega^{*} \geq \omega_{\mathrm{B}}^{*}$ including the case of $\omega^{*}=\omega_{\mathrm{B}}^{*}$ ) is not applicable to the wave motion considered in this section, even though $\Omega$ is a constant of $O(1)$. Furthermore, the weak nonuniformity of the bubble number density $\delta$ provides a small correction to the propagation speed of the envelope $A$, which is similar to the case of the generalized KZK equation (Kanagawa et al., 2011a).

Combining Eqs. (47) and (54), that is, connecting the axial region of $x=O(1 / \epsilon)$ (Far-field I) to $x=O\left(1 / \epsilon^{2}\right)$ (Farfield II), yields

$$
\begin{aligned}
& i\left(\frac{\partial A}{\partial t}+v_{\mathrm{g}} \frac{\partial A}{\partial x}\right)+\frac{q}{2} \frac{\partial^{2} A}{\partial x^{2}}+\epsilon^{2} \nu_{1}|A|^{2} A \\
& \quad+\epsilon^{2}\left(i \nu_{2}+\delta \nu_{4}\right) A+\frac{v_{\mathrm{g}}}{2 k} \frac{1}{r} \frac{\partial}{\partial r}\left(r \frac{\partial A}{\partial r}\right)=0 .
\end{aligned}
$$

The extended independent-variables $t_{1}, t_{2}, x_{1}, x_{2}$, and $r_{1}$ are restored to the original independent-variables $t, x$, and $r$ via Eq. (31). Equation (58) can then be rewritten as

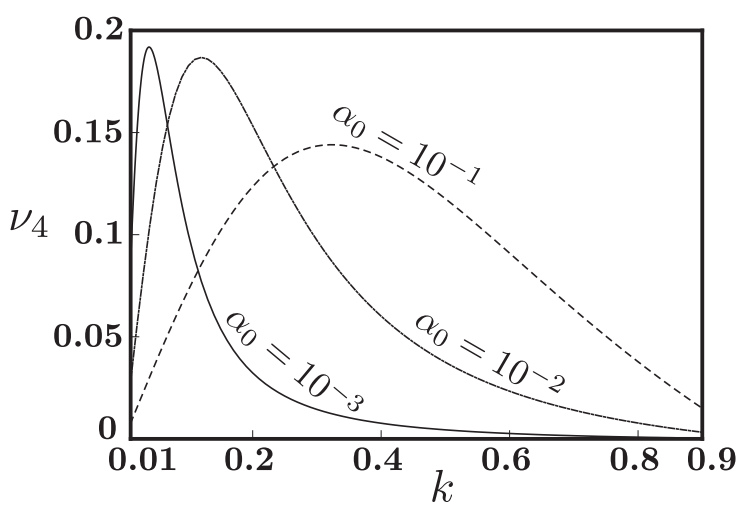

FIG. 3. The nonuniform coefficient $\nu_{4}$ [Eq. (56)] as a function of the normalized wavenumber $(0.01 \leq k \leq 0.9)$ for initial void fractions of $\alpha_{0}=10^{-1}$, $10^{-2}$, and $10^{-3}$. The initial bubble radius is fixed at $R_{0}^{*}=1 \mathrm{~mm}$, and we assume a spherical bubble oscillation of $\beta_{1}=1 / 2$, an isothermal change of the gas inside the bubble of $\gamma=1$, and the ambient conditions for the air-water system $p_{\mathrm{L} 0}^{*}=101325 \mathrm{~Pa}, \rho_{\mathrm{L} 0}^{*}=10^{3} \mathrm{~kg} / \mathrm{m}^{3}$, and $\sigma^{*}=0.0728 \mathrm{~N} / \mathrm{m}$. The normalized wave frequency $\Omega$ in Eq. (56) is dependent on $k$ and is calculated by using the linear dispersion relation $D(k, \Omega)=0$. The small wavenumber region of $k \rightarrow 0$ is outside the range of applicability of the analysis as described by Eq. (17c).

$$
\begin{aligned}
& i \frac{\partial A}{\partial X}+Q_{3} \frac{\partial^{2} A}{\partial \eta^{2}}+Q_{1}|A|^{2} A \\
& \quad+i Q_{2} A+\delta Q_{4} A+\frac{\Gamma^{2}}{2 k} \frac{1}{\zeta} \frac{\partial}{\partial \zeta}\left(\zeta \frac{\partial A}{\partial \zeta}\right)=0
\end{aligned}
$$

via the variable transformation

$$
X \equiv \epsilon^{2} x, \quad \eta \equiv \epsilon\left(t-x / v_{\mathrm{g}}\right), \quad \zeta \equiv \epsilon \Gamma r .
$$

Here, the coefficients are given by

$$
\begin{aligned}
& Q_{1}=\frac{\nu_{1}}{v_{\mathrm{g}}} \leq 0, \\
& Q_{2}=\frac{\nu_{2}}{v_{\mathrm{g}}} \geq 0, \\
& Q_{3}=\frac{q}{2 v_{\mathrm{g}}{ }^{3}} \leq 0, \\
& Q_{4}=\frac{\nu_{4}}{v_{\mathrm{g}}}
\end{aligned}
$$

Equation (59) is the generalized NLS equation, where the second, third, and fourth terms, with coefficients of $Q_{3}, Q_{1}$, and $Q_{2}$ are the strong dispersion, weak (cubic) nonlinear, and weak dissipation contributions, respectively, as in the original NLS equation (Kanagawa et al., 2010). The fifth term with the coefficient of $\delta Q_{4}$ describes the weak nonuniformity of the bubble number density $\delta$, and the nonuniform coefficient $\nu_{4}$ provides a small correction to the group velocity of the envelope $A$. The sixth term denoted by a Laplacian with respect to the normalized radial coordinate $\zeta$ describes the weak diffraction effect of the envelope.

The generalized NLS equation governs the nonlinear propagation of an envelope of diffracted sound beams (carrier waves) in the short-wavelength regime in a nonuniform bubbly liquid with dissipation and dispersion appearing in the far field. The propagation is characterized by $X, \eta$, and $\zeta$, where the temporal, axially spatial, and radially spatial scales are of $O\left(1 / \epsilon^{2}\right), O\left(1 / \epsilon^{2}\right)$, and $O(1 / \epsilon)$, respectively.

\section{GENERALIZED KZK EQUATION}

We review briefly the generalized KZK equation (Kanagawa et al., 2011a; Yano et al., 2013) and highlight the differences between the generalized NLS and KZK equations, with a focus on the size of the dispersion effect. Note that the derivation in this section only involves real constants and functions. Based on the scaling relation in Eqs. (18) and (24) for the generalized KZK equation, we derive a linear set of equations to leading order of the approximation. The difference between the linear set in Sec. IV A and that derived in this section is the form of linearized Keller equation [see Eqs. (34)-(38) in Sec. 3.1 and Eqs. (60)-(64) in Sec. 4.1 of Kanagawa et al., 2010]. This is a consequence of the parameter scaling in Eq. (18) and results in weaker dispersion compared to that of the wave in Sec. IV [Eq. (17)]. The 
dispersion term in the first-order equation (42) was originated from the presence of the inertial term in the linearized Keller equation. The wave motion considered in Sec. IV is thus strongly dispersive, while that considered in this section is weakly dispersive.

Combining the linear equations into a single equation results in the nondispersive linear wave equation for $R_{1}$,

$$
\frac{\partial^{2} R_{1}}{\partial t_{0}^{2}}-v_{\mathrm{p}}^{2} \frac{\partial^{2} R_{1}}{\partial x_{0}^{2}}=0
$$

This indicates that dissipation, dispersion, diffraction, and nonuniform effects do not appear in the near field. Here, the phase velocity $v_{\mathrm{p}}$ is given by

$$
v_{\mathrm{p}}=\sqrt{\frac{3 \alpha_{0}\left(1-\alpha_{0}+\beta_{1}\right) \gamma p_{\mathrm{G} 0}+\beta_{1}\left(1-\alpha_{0}\right) \Delta^{2} / \Omega^{2}}{3 \beta_{1} \alpha_{0}\left(1-\alpha_{0}\right)}} .
$$

Like the classical speed of sound in bubbly liquids (van Wijngaarden, 1968, 1972), $v_{\mathrm{p}}$ is also proportional to $1 / \sqrt{\alpha_{0}\left(1-\alpha_{0}\right)}$. Hereafter, we again focus on the rightrunning wave with a phase function of

$$
\phi_{0} \equiv t_{0}-x_{0}
$$

Choosing $R_{1} \equiv f\left(\phi_{0} ; t_{1}, x_{1}, r_{1 / 2}\right)$ reduces Eq. (61) to

$$
\mathcal{L}[f] \equiv \frac{\partial f}{\partial t_{0}}+\frac{\partial f}{\partial x_{0}}=0 .
$$

As a counterpart to Eq. (48) in Sec. IV B, the momentum conservation equations in the $r$ direction provide the radial variations of the velocities. That is, $v_{\mathrm{G} 1}, v_{\mathrm{L} 1}$, and derivatives with respect to $r_{1 / 2}$ appear in the approximation of $O\left(\epsilon^{3 / 2}\right)$,

$$
\frac{\partial v_{\mathrm{G} 1}}{\partial \phi_{0}}=C_{1} \Gamma \frac{\partial f}{\partial r_{1 / 2}}, \quad \frac{\partial v_{\mathrm{L} 1}}{\partial \phi_{0}}=C_{2} \Gamma \frac{\partial f}{\partial r_{1 / 2}},
$$

with

$$
\begin{aligned}
& C_{1}=\frac{3\left(1-\alpha_{0}+\beta_{1} \alpha_{0}\right)}{1-\alpha_{0}+\beta_{1}}-\frac{\left(1-\alpha_{0}\right)^{2} \Delta^{2} / \Omega^{2}}{\alpha_{0}\left(1-\alpha_{0}+\beta_{1}\right)}, \\
& C_{2}=\frac{3 \beta_{1} \alpha_{0}+\left(1-\alpha_{0}\right) \Delta^{2} / \Omega^{2}}{1-\alpha_{0}+\beta_{1}} .
\end{aligned}
$$

The velocities $v_{\mathrm{G} 1}$ and $v_{\mathrm{L} 1}$ as thus given as functions of $r_{1 / 2}$ and $\phi_{0}$.

The principal difference between the generalized NLS and KZK equations is the size of the dispersion. In the case of the weaker dispersion for the KZK equation, the focus is on the behavior of the carrier wave (rather than the envelope), and the second order of the approximation leads to a nonlinear wave equation for the far field, in which the weak dispersion competes with the weak dissipation, diffraction, and nonuniform effects, as well as a weak (second) nonlinear effect.

The set of inhomogeneous equations of $O\left(\epsilon^{2}\right)$ reduces to a single equation for $R_{2}$,

$$
\begin{aligned}
\mathcal{L}\left[R_{2}\right]= & -2 \frac{\partial}{\partial \phi_{0}}\left\{\frac{\partial f}{\partial t_{1}}+\frac{\partial f}{\partial x_{1}}+\left[\Lambda_{0}+\Lambda_{4} \delta\left(x_{1}\right)\right]\right. \\
& \left.\times \frac{\partial f}{\partial \phi_{0}}+\Lambda_{1} f \frac{\partial f}{\partial \phi_{0}}+\Lambda_{2} \frac{\partial^{2} f}{\partial \phi_{0}^{2}}+\Lambda_{3} \frac{\partial^{3} f}{\partial \phi_{0}^{3}}\right\} \\
& +\frac{\Gamma^{2}}{r_{1 / 2}} \frac{\partial}{\partial r_{1 / 2}}\left(r_{1 / 2} \frac{\partial f}{\partial r_{1 / 2}}\right)
\end{aligned}
$$

and we impose the solvability condition

$$
J\left(f ; \phi_{0}, t_{1}, x_{1}, r_{1 / 2}\right)=0 .
$$

This far field equation describes the wave motion in the axial region of $x=O(1 / \epsilon)$.

Combining the near and far field representations [i.e., Eqs. (63) and (67)] yields

$$
\begin{aligned}
& \frac{\partial}{\partial \eta}\left(\frac{\partial f}{\partial X}+\Lambda_{1} f \frac{\partial f}{\partial \eta}+\Lambda_{2} \frac{\partial^{2} f}{\partial \eta^{2}}+\Lambda_{3} \frac{\partial^{3} f}{\partial \eta^{3}}\right) \\
& \quad=\frac{\Gamma^{2}}{2 \zeta} \frac{\partial}{\partial \zeta}\left(\zeta \frac{\partial f}{\partial \zeta}\right)
\end{aligned}
$$

via the variable transformation

$$
\begin{aligned}
& X \equiv \epsilon x, \quad \eta \equiv t-\left\{1+\epsilon\left[\Lambda_{0}+\Lambda_{4} \delta(\epsilon x)\right]\right\} x, \\
& \zeta \equiv \sqrt{\epsilon} \Gamma r
\end{aligned}
$$

with coefficients of

$$
\begin{aligned}
& \Lambda_{0}=\frac{\left(1-\alpha_{0}\right) \Delta^{2} V^{2}}{6 \alpha_{0} \Omega^{2}}=-\Pi_{0}, \\
& \Lambda_{1}=-\Pi_{1}, \\
& \Lambda_{2}=-\frac{1}{6 \alpha_{0}}\left(4 \mu+\frac{\Delta^{3} V}{\Omega^{2}}\right)=\Pi_{2}, \\
& \Lambda_{3}=-\frac{\Delta^{2}}{6 \alpha_{0}}=-\Pi_{3}, \\
& \Lambda_{4}=\frac{\left[\beta_{1}+\left(1-\alpha_{0}\right)^{2}\right] \Delta^{2} / \Omega^{2}-3 \beta_{1} \alpha_{0}^{2}}{6 \alpha_{0}\left(1-\alpha_{0}\right)\left(1-\alpha_{0}+\beta_{1}\right)} .
\end{aligned}
$$

Here, $\Pi_{i}(i=0,1,2,3)$ are the coefficients in the original KdVB equation and are shown explicitly in Eqs. (52)-(54) in Sec. 3.2 and Eq. (A.1) in Appendix 2 of Kanagawa et al. (2010); the negative sign [i.e., $\Lambda_{i}=-\Pi_{i}(i=0,1,3)$ ] is caused by different definitions of the phase functions in Eqs. (62) and (68b). Equation (68) is the generalized KZK (or KP) equation. In a similar manner to the generalized NLS equation [Eq. (59a)], the second, third, and fourth terms on the left-hand side of Eq. (68a) represent the weak nonlinearity, weak dissipation, and weak dispersion, respectively, as in the case of the original $\mathrm{KdVB}$ equation (Kanagawa et al., 2010). The right-hand side of Eq. (68a) describes the weak diffraction effect. The weak nonuniformity of the bubble number density, $\delta$, provides a small 
correction to the phase velocity through the retarded time $\eta$ in Eq. (68b).

To discuss the nonuniform effect, we examine the dependence of $\Lambda_{4}$ on $\alpha_{0}$. In this case, $\Lambda_{4}$ is positive for the air-water system under ambient conditions, and this is true even for the moderately large initial void fraction of $\alpha_{0}=0.1$, which is within the applicable range of the present analysis as stated in Sec. III D. Similar to the case of the nonuniform coefficient $\nu_{4}$ in Sec. IV C, the dependence of $\Lambda_{4}$ on $R_{0}^{*}$ is negligibly small.

The generalized KZK equation governs the nonlinear propagation of diffracted sound beams that are subject to dissipation and dispersion in a nonuniform bubbly liquid in a field characterized by $X, \eta$, and $\zeta$, where the temporal, axially spatial, and radially spatial scales are of $O(1 / \epsilon), O(1 / \epsilon)$, and $O(1 / \sqrt{\epsilon})$, respectively.

\section{CONCLUSIONS}

We have successfully derived two types of nonlinear wave equations for weakly nonlinear propagation of diffractive sound beams in nonuniform bubbly liquids by extending an earlier theory (Kanagawa et al., 2010) for plane wave propagation in uniform bubbly liquids. This was accomplished in two steps: (i) by adding a scaling relation of the typical diameter of the beam, $L^{*} / D^{*}$, with respect to the nondimensional wave amplitude $\epsilon(\ll 1)$, and (ii) by assuming that the initial nonuniformity of the bubble number density and its spatial variation were as small as the wave amplitude $\epsilon$. The former introduced weak diffraction of the beam, while the latter introduced weak nonuniformity of the bubble number density. The essence is summarized as

$$
\left(\frac{U^{*}}{c_{\mathrm{L} 0}^{*}}, \frac{\omega^{*}}{\omega_{\mathrm{B}}^{*}}, \frac{R_{0}^{*}}{L^{*}}, \frac{L^{*}}{D^{*}}\right) \equiv\left(O\left(\epsilon^{a}\right), O\left(\epsilon^{b}\right), O\left(\epsilon^{c}\right), O\left(\epsilon^{d}\right)\right),
$$

with

$$
\begin{cases}a=2, b=c=0, d=1 & \text { (for NLS), } \\ a=b=c=d=1 / 2 & \text { (for KZK). }\end{cases}
$$

The incorporation of real number $d$ [i.e., the scaling of $O\left(\epsilon^{d}\right)$ ] in Eqs. (70) and (71) that describes the relative size of the diffraction to the nonlinearity, yields the progress in the theory from Eq. (1).

Based on these relationships, we succeeded in deriving, from the basic equations for bubbly flows in the two-fluid model, a generalized NLS equation including dissipation, diffraction, and nonuniform effects for short-wavelength high-frequency waves, and a generalized KZK (KP) equation including dispersion (dissipation) term and nonuniform effect for long-wavelength low-frequency waves. These nonlinear wave equations govern the long-range propagation of diffractive sound beams in nonuniform bubbly liquids with dissipation and dispersion effects. Neither of these equations had ever been derived from the two-fluid model in the context of bubbly flow theory. The generalized NLS and KZK equations describe the most simple balance (i.e., linear combination) of dissipation, dispersion, diffraction, and nonlinearity in nonuniform bubbly liquids. This paper will encourage further studies such as the introduction of an interaction between nonlinearity and diffraction.

\section{ACKNOWLEDGMENTS}

The author wishes to thank Dr. Ryu Egashira for his helpful comments and Junya Kawahara for fruitful discussions. The present work was supported by the Japan Society for the Promotion of Science (JSPS) KAKENHI (Grant Nos. 245892 and 26820040).

\section{APPENDIX: INHOMOGENEOUS TERMS}

The explicit expressions of $\tilde{N}_{i}(i=1,2,3,4,5)$ that make up the forms of the inhomogeneous terms in Eq. (51) are given by

$$
\begin{aligned}
& \tilde{N}_{1}=-\Gamma \frac{1}{r_{1}} \frac{\partial r_{1} v_{\mathrm{G} 1}}{\partial r_{1}}+\delta\left(-\frac{\partial u_{\mathrm{G} 1}}{\partial x_{0}}+3 \frac{\partial R_{1}}{\partial t_{0}}\right)+N_{1}, \\
& \tilde{N}_{2}=\Gamma\left(1-\alpha_{0}\right) \frac{1}{r_{1}} \frac{\partial r_{1} v_{\mathrm{L} 1}}{\partial r_{1}}-\delta \alpha_{0} \frac{\partial u_{\mathrm{L} 1}}{\partial x_{0}}+N_{2}, \\
& \tilde{N}_{3}=\delta\left[-\beta_{1} \frac{\partial}{\partial t_{0}}\left(u_{\mathrm{G} 1}-u_{\mathrm{L} 1}\right)+3 \gamma p_{\mathrm{G} 0} \frac{\partial R_{1}}{\partial x_{0}}\right]+N_{3}, \\
& \tilde{N}_{4}=\delta\left[\beta_{1} \alpha_{0} \frac{\partial}{\partial t_{0}}\left(u_{\mathrm{G} 1}-u_{\mathrm{L} 1}\right)+\alpha_{0} \frac{\partial u_{\mathrm{L} 1}}{\partial t_{0}}+\alpha_{0} \frac{\partial p_{\mathrm{L} 1}}{\partial x_{0}}\right]+N_{4}, \\
& \tilde{N}_{5}=N_{5},
\end{aligned}
$$

where $N_{i}(i=1,2,3,4,5)$ are presented in Appendix 1 of Kanagawa et al. (2010).

In addition, the inhomogeneous terms in Eq. (52), $S_{1}$ and $S_{2}$, are given by

$$
\begin{aligned}
S_{1}= & -\beta_{1} \alpha_{1} \frac{\partial}{\partial t_{0}}\left(v_{\mathrm{G} 1}-v_{\mathrm{L} 1}\right)-\beta_{1} \frac{\partial}{\partial t_{1}}\left(v_{\mathrm{G} 1}-v_{\mathrm{L} 1}\right) \\
& -\beta_{1}\left(u_{\mathrm{G} 1} \frac{\partial v_{\mathrm{G} 1}}{\partial x_{0}}-u_{\mathrm{L} 1} \frac{\partial v_{\mathrm{L} 1}}{\partial x_{0}}\right)-\beta_{2}\left(v_{\mathrm{G} 1}-v_{\mathrm{L} 1}\right) \frac{\partial \alpha_{1}}{\partial t_{0}} \\
& -\Gamma p_{\mathrm{G} 0} \frac{\partial}{\partial r_{1}}\left[-3 \gamma R_{2}+\frac{3 \gamma(3 \gamma+1)}{2} R_{1}^{2}\right] \\
& +3 \Gamma \gamma p_{\mathrm{G} 0} \alpha_{1} \frac{\partial R_{1}}{\partial r_{1}} \\
S_{2}= & \beta_{1} \alpha_{0} \alpha_{1} \frac{\partial}{\partial t_{0}}\left(v_{\mathrm{G} 1}-v_{\mathrm{L} 1}\right)+\beta_{1} \alpha_{0} \frac{\partial}{\partial t_{1}}\left(v_{\mathrm{G} 1}-v_{\mathrm{L} 1}\right) \\
& +\beta_{1} \alpha_{0}\left(u_{\mathrm{G} 1} \frac{\partial v_{\mathrm{G} 1}}{\partial x_{0}}-u_{\mathrm{L} 1} \frac{\partial v_{\mathrm{L} 1}}{\partial x_{0}}\right) \\
& +\beta_{2} \alpha_{0}\left(v_{\mathrm{G} 1}-v_{\mathrm{L} 1}\right) \frac{\partial \alpha_{1}}{\partial t_{0}} \\
& -\left(1-\alpha_{0}\right) \frac{\partial v_{\mathrm{L} 1}}{\partial t_{1}}-\left(1-\alpha_{0}\right) \frac{\partial u_{\mathrm{L} 1} v_{\mathrm{L} 1}}{\partial x_{0}}+\alpha_{0} \frac{\partial \alpha_{1} v_{\mathrm{L} 1}}{\partial t_{0}} \\
& +\Gamma \alpha_{0} \alpha_{1} \frac{\partial p_{\mathrm{L} 1}}{\partial r_{1}}-\Gamma\left(1-\alpha_{0}\right) \frac{\partial p_{\mathrm{L} 2}}{\partial r_{1}}+\Gamma \alpha_{0} \Delta^{2} \Omega^{2} R_{1} \frac{\partial \alpha_{1}}{\partial r_{1}}
\end{aligned}
$$


Akhatov, I., Parlitz, U., and Lauterborn, W. (1996). "Towards a theory of self-organization phenomena in bubble-liquid mixtures," Phys. Rev. E 54, 4990-5003.

Akhatov, I. Sh., and Khismatullin, D. B. (2001). "Mechanisms of interaction between ultrasound and sound in liquids with bubbles: Singular focusing," Acoust. Phys. 47, 178-182.

Ando, K., Colonius, T., and Brennen, C. E. (2011). "Numerical simulation of shock propagation in a polydisperse bubbly liquid," Int. J. Multiphase Flow 37, 596-608.

Bailey, M. R., Khokhlova, V. A., Sapozhnikov, O. A., Kargl, S. G., and Crum, L. A. (2003). "Physical mechanisms of the therapeutic effect of ultrasound (a review)," Acoust. Phys. 49, 369-388.

Blackstock, D. T. (2000). "Radiation from a baffled piston," in Fundamentals of Physical Acoustics (Wiley-Interscience, New York), Chap. 13, pp. 440-464.

Commander, K. W., and Prosperetti, A. (1989). "Linear pressure waves in bubbly liquids: Comparison between theory and experiments," J. Acoust. Soc. Am. 85, 732-746.

Drew, D. A. (1983). "Mathematical modelling of two-phase flow," Ann. Rev. Fluid Mech. 15, 261-291.

Eames, I., and Hunt, J. C. R. (2004). "Forces on bodies moving unsteadily in rapidly compressed flows," J. Fluid Mech. 505, 349-364.

Egashira, R., Yano, T., and Fujikawa, S. (2004). "Linear wave propagation of fast and slow modes in mixtures of liquid and gas bubbles," Fluid Dyn. Res. 34, 317-334.

Grandjean, H., Jacques, N., and Zaleski, S. (2012). "Shock propagation in liquids containing bubble clusters: A continuum approach,” J. Fluid Mech. 701, 304-332.

Gumerov, N. A. (1992). "Quasi-monochromatic weakly non-linear waves in a low dispersion bubble medium," J. Appl. Math. Mech. 56, 50-59.

Hamilton, M. F. (1998). "Sound beams," in Nonlinear Acoustics, edited by M. F. Hamilton and D. T. Blackstock (Academic Press, San Diego), Chap. 8, pp. 233-262.

Jeffrey, A., and Kawahara, T. (1982). Asymptotic Methods in Nonlinear Wave Theory (Pitman Publishing, Boston).

Kadomtsev, B. B., and Petviashvili, V. I. (1970). "On the stability of solitary waves in weakly dispersing media," Sov. Phys. Acoust. 15, 539-541.

Kanagawa, T., Watanabe, M., Yano, T., and Fujikawa, S. (2011b). "Nonlinear wave equations for pressure wave propagation in liquids containing gas bubbles (comparison between two-fluid model and mixture model)," J. Fluid Sci. Technol. 6(6), 838-850.

Kanagawa, T., Yano, T., Watanabe, M., and Fujikawa, S. (2010). "Unified theory based on parameter scaling for derivation of nonlinear wave equations in bubbly liquids," J. Fluid Sci. Technol. 5(3), 351-369.

Kanagawa, T., Yano, T., Watanabe, M., and Fujikawa, S. (2011a). "Nonlinear wave equation for ultrasound beam in nonuniform bubbly liquids,” J. Fluid Sci. Technol. 6(2), 279-290.

Keller, J. B., and Kolodner, I. I. (1956). "Damping of underwater explosion bubble oscillations," J. Appl. Phys. 27, 1152-1161.

Khismatullin, D. B., and Akhatov, I. Sc. (2001). "Sound-ultrasound interaction in bubbly fluids: Theory and possible applications," Phys. Fluids 13, 3582-3598.
Kudryashov, N. A., and Sinelshchikov, D. I. (2013). "An extended equation for the description of nonlinear waves in a liquid with gas bubbles," Wave Motion 50, 351-362.

Kudryashov, N. A., and Sinelshchikov, D. I. (2014). "Extended models of non-linear waves in liquid with gas bubbles," Int. J. Nonlinear. Mech. 63, 31-38.

Kuznetsov, V. P. (1971). "Equation of nonlinear acoustics," Sov. Phys. Acoust. 16, 467-470.

Kuznetsov, V. V., Nakoryakov, V. E., Pokusaev, B. G., and Shreiber, I. R. (1978). "Propagation of perturbations in a gas-liquid mixture," J. Fluid Mech. 85, 85-96.

Leroy, V., Strybulevych, A., Page, J. H., and Scanlon, M. G. (2011). "Influence of positional correlations on the propagation of waves in a complex medium with polydisperse resonant scatterers," Phys. Rev. E 83, 046605.

Liang, B., Zou, X. Y., and Cheng, J. C. (2008). "Effective medium method for sound propagation in a soft medium containing air bubbles," J. Acoust. Soc. Am. 124, 1419-1429.

Louisnard, O. (2012). "A simple model of ultrasound propagation in a cavitating liquid. Part I: Theory, nonlinear attenuation and traveling wave generation," Ultrason. Sonochem. 19, 56-65.

Nakoryakov, V. E., Pokusaev, B. G., and Shreiber, I. R. (1993). Wave Propagation in Gas-Liquid Media, 2nd ed. (CRC Press, Heidelberg).

Nayfeh, A. H. (1973). Perturbation Methods (Wiley-Interscience, New York).

Nigmatulin, R. I. (1991). Dynamics of Multiphase Media (Hemisphere, New York), Vol. 2

Prosperetti, A. (1991). "The thermal behaviour of oscillating gas bubbles," J. Fluid Mech. 222, 587-616.

Prosperetti, A., and Jones, A. V. (1984). "Pressure forces in disperse twophase flow," Int. J. Multiphase Flow 10(4), 425-440.

Vanhille, C., and Campos-Pozuelo, C. (2009). "Nonlinear ultrasonic waves in bubbly liquids with nonhomogeneous bubble distribution: Numerical experiments," Ultrason. Sonochem. 16, 669-685.

van Wijngaarden, L. (1968). "On the equations of motion for mixtures of liquid and gas bubbles," J. Fluid Mech. 33, 465-474.

van Wijngaarden, L. (1972). "One-dimensional flow of liquids containing small gas bubbles,” Ann. Rev. Fluid Mech. 4, 369-394.

Watanabe, M., and Prosperetti, A. (1994). "Shock waves in dilute bubbly liquids," J. Fluid Mech. 274, 349-381.

Whitham, G. B. (1974). Linear and Nonlinear Waves (Wiley, New York).

Yano, T., Egashira, R., and Fujikawa, S. (2006). "Linear analysis of dispersive waves in bubbly flows based on averaged equations," J. Phys. Soc. Jpn. 75, 104401-104408.

Yano, T., Kanagawa, T., Watanabe, M., and Fujikawa, S. (2013). "Nonlinear wave propagation in bubbly liquids," in Bubble Dynamics and Shock Waves, edited by Can F. Delale, Shock Wave Science and Technology Reference Library (Springer, Heidelberg), Vol. 6, Chap. 4, pp. 107-140.

Zabolotskaya, E. A., and Khokhlov, R. V. (1969). "Quasi-plane waves in the non-linear acoustics of confined beams," Sov. Phys. Acoust. 15, $35-40$

Zemanek, J. (1971). "Beam behavior within the nearfield of a vibrating piston,” J. Acoust. Soc. Am. 49, 181-191.

Zhang, D. Z., and Prosperetti, A. (1994). "Averaged equations for inviscid disperse two-phase flow," J. Fluid Mech. 267, 185-219. 